\title{
Multimodal Characterization of Hierarchically Porous Nanocomposite Materials: The Case Study of the PEARL Membrane
}

Stephanie Ribet ${ }^{1}$, Benjamin Shindel ${ }^{2}$, Roberto dos Reis ${ }^{2}$, Vikas Nandwana ${ }^{3}$ and Vinayak Dravid ${ }^{2}$

${ }^{1}$ Evanston, Illinois, United States, ${ }^{2}$ Department of Materials Science and Engineering, Northwestern

University, United States, ${ }^{3}$ Department of Materials Science and Engineering, Northwestern University, Evanston, Illinois, United States

It is well known that it can be challenging to characterize hybrid materials via electron microscopy due to the inherent mismatch in mechanical properties between their hard and soft components and because of the dose limitations imposed by the electron beam-sensitive constituents.[1-3] This challenge can be exacerbated by the unusual geometries of real-world samples, requiring correlative study and creative sample preparation. Nonetheless study of these hybrid structures and their form-function relationships allows for enhanced biological understanding and improved design rules for technological innovations.

We have developed an environmental remediation approach based on dip-coating a porous substrate (sponge or membrane) with a slurry of multifunctional nanostructures (MNS) with tailored affinity for pollutants. [4,5] Our Phosphate Elimination $\underline{\text { And }}$ Recovery Lightweight (PEARL) membrane, made by coating cellulose with iron oxide nanoparticles, can selectively sequester $>99 \%$ of phosphates from polluted waters. Mild tuning of $\mathrm{pH}$ leads to atwill adsorption and desorption, allowing for nutrient recovery and reuse of the membrane for many cycles. The PEARL membrane is an archetypical example of these aforementioned complex hybrid structures. In addition to being comprised of a hard and soft component, it is hierarchically porous, meaning it needs investigation at multiple length scales.

Figure 1a shows the typical hierarchically porous architecture of cellulose at the micro scale with scanning electron microscopy (SEM). Evaluating such a structure is difficult because of the inconsistent beam-specimen interactions with a porous structure and because the cellulose-nanoparticle interface is buried inaccessibly beneath the surface. Thus, we embedded the sponge in resin and used an ultramicrotome approach to visualize the PEARL membrane, as shown in Figure 1b. Backscattered electrons, carrying compositional information, highlight the location of the iron-oxide coating. Because the molecular scale phosphate-nanoparticle interactions happen at the membrane's solid-fluid interface, these SEM images suggest that the nanocoating was successfully applied, as the functional component is present only at the cellulose surface.

A multimodal scanning transmission electron microscopy (STEM) approach was employed to investigate more closely regions with low levels of coating (marked in yellow in 1b). The bright field image (1c), dark field image (1d), and energy-dispersive X-ray spectroscopy (EDS) map (1e) from the yellow region in 1b show an ultrathin coating in areas that at the microscale did not seem to have nanoparticles present.

Thermogravimetric analysis of the PEARL membrane is shown in Figure 1f, indicating that the coating represents $5-10 \%$ of the structure mass. This data highlights how little coating is needed to transform cellulose into an effective remediation tool. The linear isotherms displayed in Figure 1g reveal the surface area of the nanoparticles alone, cellulose alone, and the nanocomposite structure. The combined TGA and isotherm information demonstrates how efficiently the nanoparticle active material is loaded on the cellulose.

In addition to developing an understanding of the PEARL membrane, we are interested in the mechanism of nanoparticle-pollutant interactions. We prepared samples of MNS alone for X-ray photoelectron spectroscopy (XPS) and S/TEM analysis. Figure 2a-c shows iron, oxygen, and phosphorus XPS curves, which provide insight into how the electronic state of the nanoparticles change after the first cycle of adsorption. 
Figure 2d-e shows a HR-TEM image and corresponding SAED pattern of a typical area of a study. Despite the intrinsic challenges in detecting phosphorus edges with EELS in this system,[6] with the help of a direct electron detector and a nearest neighbor averaging algorithm to improve signal-to-noise, we are able to measure phosphorus's L edge and iron's M edge to confirm nanoscale binding. Ongoing investigations are looking at mapping these edges and their fine structure spatially to further understand the mechanism of iron-phosphate interaction.

The extensive characterization of the PEARL membrane provides compelling scientific insights into the binding of phosphates and suggests how this approach can be tailored for other pollutants. Using the PEARL membrane as an example, this presentation will demonstrate how proper sample preparation and correlative, multimodal characterization can be used to establish structure-property relationships in diverse materials with hard-soft interfaces.[7]
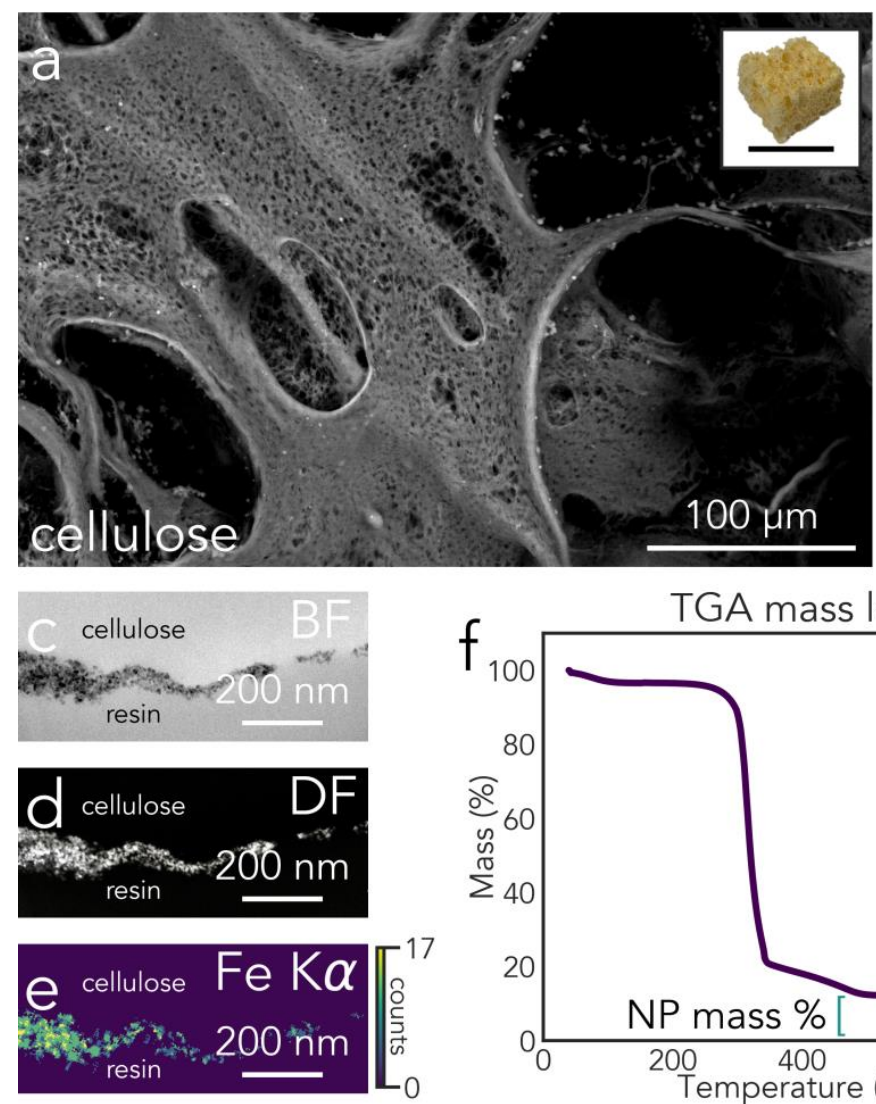
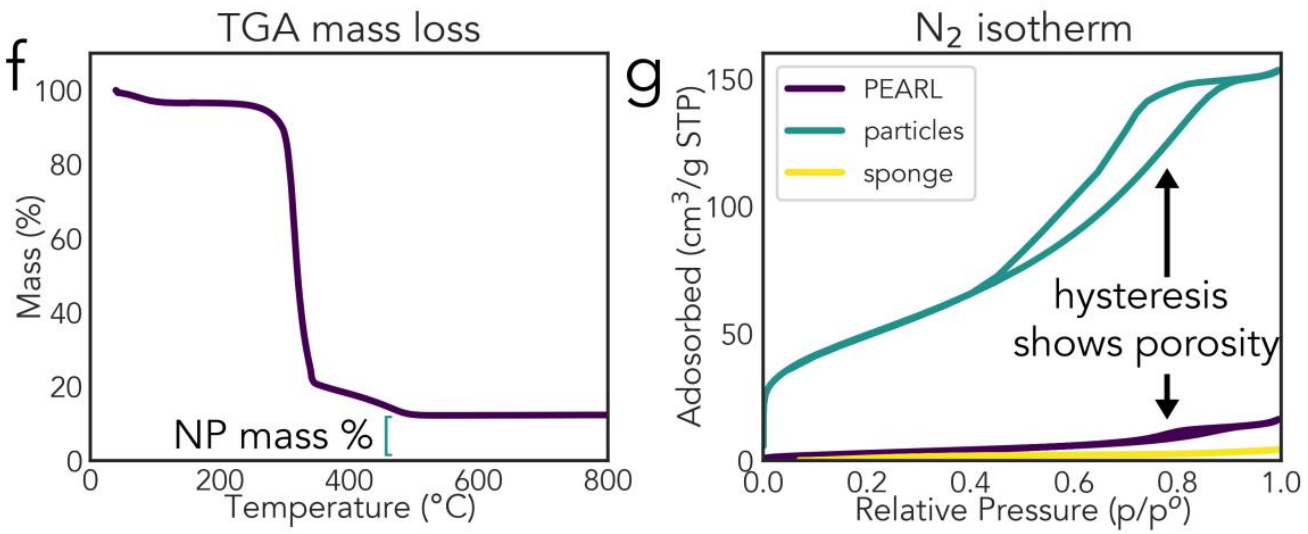

Figure 1. (a) Cellulose imaged at the microscale. Inset shows bulk cellulose. (b) SEM image of cross section of the PEARL membrane reveals nature of the coating. Inset shows PEARL membrane. (c) BF, (d) DF, and (e) EDS map from area marked in yellow in (b) shows an ultrathin iron oxide nanoparticle coating. ( $f$ ) TGA quantifies the mass loading of nanoparticles on the PEARL membrane. (g) N2 adsorption isotherm reveals surface area and porosity profile of PEARL membrane, cellulose, and nanoparticles. Inset scale bar in (a) and (b) is $2 \mathrm{~cm}$. 

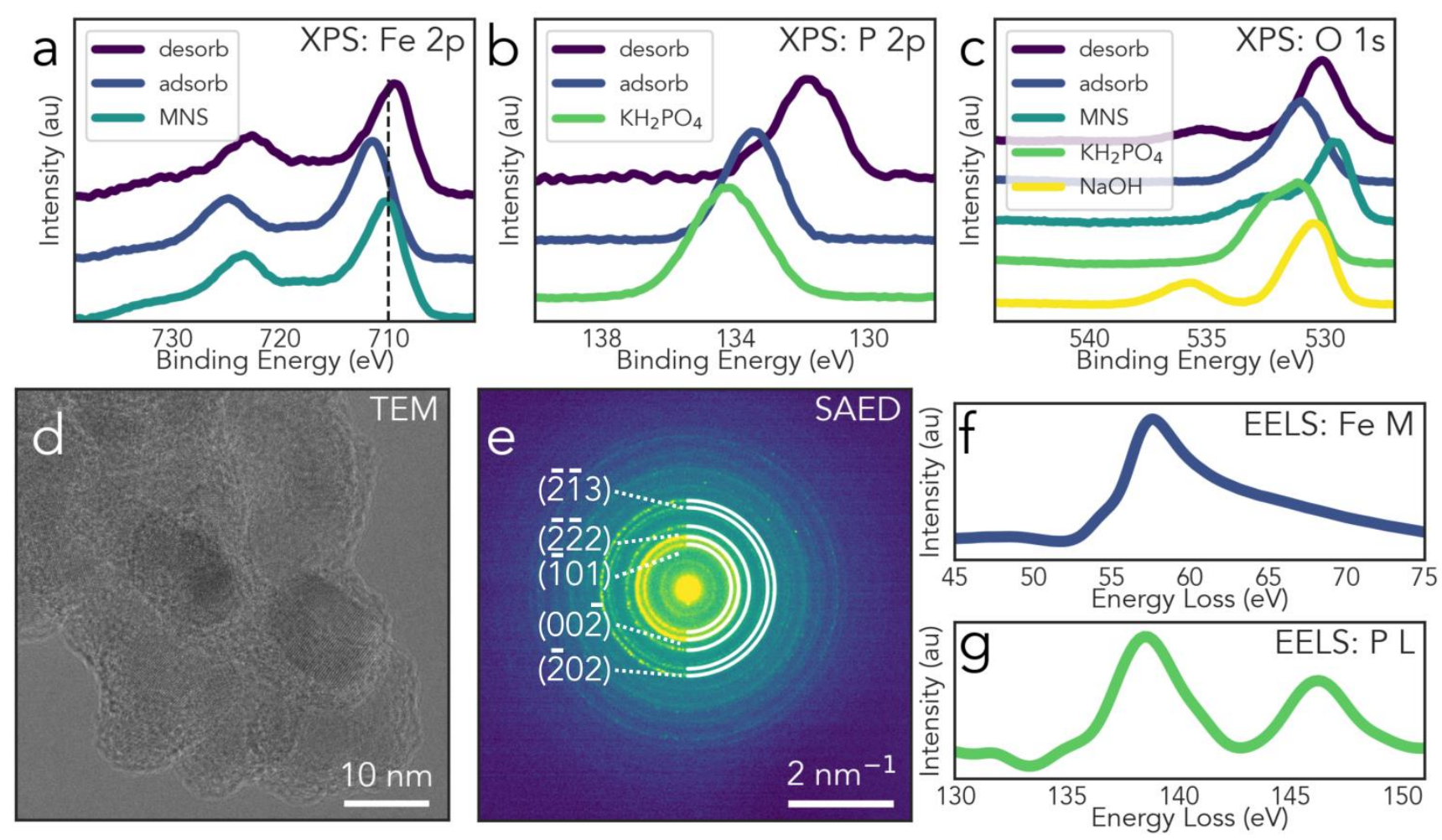

Figure 2. XPS curves showing (a) iron (b) phosphorus and (c) oxygen electronic state of multifunctional nanostructures (MNS) alone, after adsorption (adsorb), and after desorption (desorb). $\mathrm{NaOH}$ and $\mathrm{KH} 2 \mathrm{PO} 4$ shown as reference. Line in (a) added to aid the eye. (d) High resolution TEM image and corresponding (e) selected area electron diffraction (SAED) pattern show MNS structure and composition. (f) Iron and (g) phosphorus EELS edges confirm binding on the nanoscale.

\section{References}

[1] Egerton, R. F. (2019). Radiation damage to organic and inorganic specimens in the TEM. Micron, 119, $72-87$.

[2] Mayer, J., Giannuzzi, L. A., Kamino, T., \& Michael, J. (2007). TEM sample preparation and FIB-induced damage. MRS bulletin, 32(5), 400-407.

[3] Ribet, S.M., Murthy, A. A., Roth, E. W., Reis, R. D., \& Dravid, V. P. (2021). Making the Most of your Electrons: Challenges and Opportunities in Characterizing Hybrid Interfaces with STEM. Materials Today. Invited review article, under review.

[4] Nandwana, V., Ribet, S. M., Reis, R. D., Kuang, Y., More, Y., \& Dravid, V. P. (2020). OHM Sponge: A Versatile, Efficient, and Ecofriendly Environmental Remediation Platform. Industrial \& Engineering Chemistry Research, 59(23), 10945-10954.

[5] Ribet, S.M., Shindel, B., Reis, R.D., Nandwana, V., \& Dravid, V.P. (2021). Phosphate Elimination and Recovery Lightweight (PEARL) Membrane: A Sustainable Environmental Remediation Approach. Under review.

[6] Sousa, A. A., \& Leapman, R. D. (2012). Development and application of STEM for the biological sciences. Ultramicroscopy, 123, 38-49.

[7] The research related to oxide nanostructured architecture was initially supported by the National Science Foundation under Grant No. DMR-1929356 (Ceramics Program, PM: Dr. Lynnette Madsen). This work made use of the EPIC, BioCryo, and Keck-II facilities of Northwestern University's NUANCE Center, which has received support from the SHyNE Resource (NSF ECCS-2025633), the IIN, and Northwestern's MRSEC program (NSF DMR-1720139). Elemental analysis was performed at the 
Northwestern University Quantitative Bio-element Imaging Center. Nitrogen adsorption isotherm analysis was performed at the Northwestern Reactor Engineering and Catalyst Testing core facility. 